\title{
基于超声热波成像技术的机车钩舌的裂纹检测
}

\author{
敬甫盛 ${ }^{1}$, 李 朋 $^{1}$, 江海军 ${ }^{2}$, 陈 力 $^{2}$, 魏益兵 ${ }^{2}$ \\ （1. 神华铁路货车运输有限责任公司, 河北 沧州 062350； 2. 南京诺威尔光电系统有限公司, 江苏 南京 210046)
}

\begin{abstract}
摘要: 铁路机车钩舌部件长期受到挤压、弯曲、冲击, 造成材料疲劳, 产生细微裂纹, 裂纹在铁路机 车长期运行过程中会逐渐扩张, 容易造成重大事故, 因而对铁路机车钩舌裂纹检测十分重要。但铁路 机车钩舌部件表面布满铁锈、灰尘、油污等，常规无损检测技术手段无法直接应用，本文采用超声热 波成像技术对机车钩舌部件进行裂纹检测, 该技术对表面的形状、锈迹、粉尘及污染等不敏感, 在裂 纹等缺陷检测中有特殊的应用优势。通过该系统对机车钩舌的裂纹检测, 成功检测出钩舌裂纹缺陷, 验证了该技术对机车钩舌裂纹的检测能力。
\end{abstract}

关键词: 超声热波成像; 机车钩舌; 裂纹检测

中图分类号：U269.322 文献标识码：A 文章编号：1001-8891(2020)02-0158-05

\section{Crack Detection of Locomotive Hook Tongue Based on Ultrasonic Thermography}

\author{
JING Fusheng ${ }^{1}$, LI Peng ${ }^{1}$, JIANG Haijun ${ }^{2}$, CHEN Li ${ }^{2}$, WEI Yibing ${ }^{2}$ \\ (1. Shenhua Railway Freight Car Transportation Limited Liability Company, Cangzhou 062350, China;
}

2. Novelteq Ltd., Nanjing 210038, China)

\begin{abstract}
The hook tongue parts of railway locomotives undergo squeezing, flexing, and impaction over long time periods, causing material fatigue. Cracks in railway locomotives gradually expand over the long term, which can cause serious accidents. Thus, it is very important to detect such cracks. However, as the surface of a railway locomotive hook tongue is covered with rust, dust, oil, etc., conventional nondestructive testing technology cannot be applied directly to it. Ultrasonic thermography technology was used in this study to detect cracks in the coupler parts of locomotives. The technology was not sensitive to the shape of the surface and the rust, dust, and the other pollutants on it. The technology offers special application advantages in the detection of cracks and other defects. Crack flaws were detected successfully during the crack detection for a locomotive hook tongue, thereby verifying the detection ability of the technology.
\end{abstract}

Key words: ultrasonic thermography, hook tongue, crack detection

\section{0 引言}

铁路机车零件在加工制造过程中, 经常出现铸、 锻、热处理和机械加工产生的裂纹等缺陷。有些缺陷 隐藏在材料内部, 外观上看不出来, 或者外观上仅仅 看见很小的裂纹, 而内部缺陷很大很深。这些缺陷如 果不能及时准确地检测出来, 势必造成安全隐患。也 有零件原来完好, 但由于长期受到挤压、弯曲、冲击, 造成材料疲劳, 产生细微裂纹, 如不及时发现处理, 将很快向深处发展, 终成致命的事故。大多数关键零 部件的损毁, 最初都是一些微细裂纹, 甚至不加上很 重的压力时肉眼都无法看见。但在长期运行中, 裂纹
逐渐扩张, 有可能造成严重的后果。因此, 对各种铁 路机车钩舌进行检测, 是铁路行业一直高度重视的领 域 ${ }^{[1-4]}$ 。

在轨道车辆制造过程中, 相关的无损检测方法包 括射线、超声、磁粉、浴流、渗透等。射线检测方法 不适用于面积型缺陷的检测; 超声检测方法需要耦合 剂, 并且只能单点检测, 不能面检测; 磁粉检测适合 于检测表明近表面缺陷, 裂纹方向平行于磁力线传播 方向时, 容易发生漏检, 检测范围小, 速度慢, 工件 表面覆盖层会降低检测灵敏度; 浴流检测受趋肤效应 的限制, 多应用于工件中单点的检测; 渗透检测操作 繁琐, 容易污染环境, 同时检测成本较高。鉴于常规 
检测技术的弊端, 很难检测出轨道车辆制造加工中裂 纹缺陷。铁路机车钩舌的检测具有多重难点: 表面布 满铁锈、灰尘、油污等污染物, 常规的无损检测技术 手段无法直接应用, 必须进行清洗; 部件体积大而笨 重搬运及检测困难; 部件外形不规整, 表面粗䊅, 对 常规检测手段带来困难。轨道车辆制造过程中裂纹检 测技术的研究是当前设备监测诊断技术发展的一个 重要领域。采用常规检测技术, 很难达到高效率、低 成本、快速发现裂纹。因而必须开发出新兴检测技术。

超声热波成像技术是一种新兴的无损检测技 术 $^{[5-8]}$, 该技术对试件中的隐藏裂纹非常敏感 ${ }^{[9-13]}$; 该 技术是利用试件内部缺陷区域吸收耦合的超声能量 来产生热量, 同时采用红外热像仪记录试件表面温度 的变化, 从而得到试件内部缺陷的信息。超声激励属 于选择性的激励方式, 只在缺陷部位产生热量, 热波 在缺陷内部向试件表面传播过程中不容易受到周围 热波信号的干扰，因此表面温度信号的变化更明显， 该技术检测轨道车辆中裂纹缺陷非常具有优势。目前 在西方先进工业国家已经成功地应用在航天航空领 域的关键金属部件的裂纹缺陷检测 ${ }^{[14-16]}$, 如发动机的 叶片等。

超声热波成像技术非常适合应用于铁路车辆关 键部件的裂纹检测, 该技术对检测表面的形状、锈迹、 粉尘及污染等不敏感, 因此可能实现在役检测。超声 激励热波无损检测技术在超声耦合、热激励研究、图 像处理、系统集成等方面都需要有创新。但是该技术 目前主要应用于小体积的零件检测, 对于大体积的铁 路车辆部件上未见应用, 该技术应用于机车钩舌的裂 纹检测难点主要体现在两个方面, 其一在于如何在大 部件体内产生足够强度的混沌声场; 其二在于该技术 目前主要在实验室使用, 采用不同厂家部件组装而 成, 怎么把该技术从实验室转化到工业应用中。

\section{1 方法论述}

\section{1 基本原理}

超声热波成像技术又称为超声激励红外无损检 测技术, 超声热像成像技术是将超声激励和红外无损 检测相结合的新型无损检测技术, 属于主动式红外无 损检测方法的一种, 超声热波成像技术原理示意图如 图 1 所示。超声热波成像技术采用 $20 \mathrm{kHz}$ 的超声波耦 合进试件, 超声波在固体中传播很快, 裂纹缺陷区域 在超声波的作用下, 由于摩擦生热、塑性变形、热弹 效应等产生热量, 裂纹缺陷区域温度升高产生的热波 信号从裂纹缺陷区域向试件表面传播, 裂纹缺陷处对 应的试件表面温度比无裂纹缺陷处温度高, 通过红外
热像仪采集试件表面的热波信号, 得到了试件表面红 外热图序列。由于不同深度的裂纹所产生的红外信号 传到试件表面的时间是不同的，因此可以对红外信号 强度随时间变化的关系来对裂纹的深度、大小等信息 进行判断。

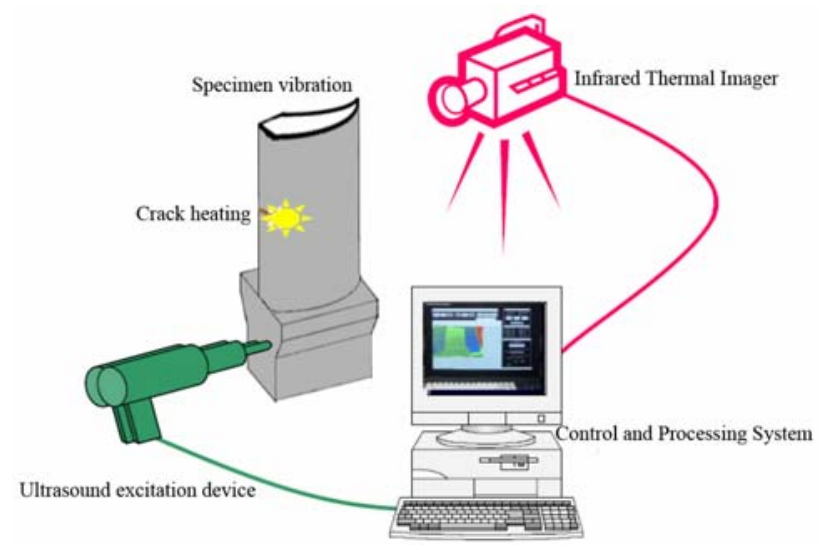

图 1 超声热波成像技术原理示意图

Fig. 1 Schematic diagram of ultrasonic thermal wave imaging technology

超声热波成像技术检测过程中热波信号的转化 过程如下:

1) 超声能量进入试件;

2) 裂纹缺陷处由于热弹效应、摩擦生热使得缺 陷区域温度升高;

3）缺陷处的温度由缺陷内部传播到试件表面;

4）红外热像仪采集试件表面的温度信号。

超声热波成像技术与其他激励方式的红外无损 检测技术相比其特点在于: 一是超声激励属于选择性 激励, 仅对缺陷区域进行加热, 非缺陷区域几乎不会 产生热量。缺陷区域与非缺陷区域的热波信号信噪比 更高, 更容易检测出缺陷。二是检测时间快, 超声在 固体中传播速度很快, 超声从激励位置传播到缺陷位 置几乎一瞬间，热波信号直接从缺陷区域传播到试件 表面，不用考虑从表面传播到缺陷区域，因而其检测 的时间非常短, 从超声激励开始到裂纹缺陷检测完成 基本在 $5 \mathrm{~s}$ 内完成。由于热波属于衰减波, 热波在传 播过程中由于热扩散容易导致热波信号的衰减, 超声 热波成像技术的上述两个优点可以很好地减弱热波 信号衰减带来的影响。因而超声热波成像技术能更好 地检测出试件内部的微小裂纹。

\section{2 技术优势}

超声热波成像技术对工件施加超声激励, 所需的 电功率并不大, 与磁粉检测所需电能相比, 只有磁粉 检测耗电的 $1 / 10$ 。这样一来, 实际制成的设备很轻很 小，可能制成便携式拿到现场运用。它的检测时间只 有几秒, 甚至可以使用电池供电。 
超声热波成像无损检测技术作为一种主动式热 激励热像成像技术, 在热激励方式与图像处理手段等 方面都有创新, 其技术主要有下述特点: 用途广, 可 用于金属和非金属材料; 与浴流检测法相比, 这种方 法不限于导电材料的检测。陶瓷、粉末冶金、注塑材 料均可检测; 速度快, 单次测量一般只需几秒钟; 单 次测面积大; 超声波能在材料中较远距离的传导, 因 而可以进行远距离热激励; 测量结果用图像显示、直 观易懂; 对裂纹型缺陷敏感, 尤其是垂直于材料表面 的细小裂纹; 该技术尤其对闭合的疲劳裂纹比较有 效, 因为这种裂纹的两壁贴合, 容易产生摩擦而发热, 而这种裂纹是其他方法比较难于检测的; 与渗透探伤 相比, 这种技术可能探测到材料内部的缺陷, 不一定 裂纹要扩展到表面才被渗透探伤方式检测出来。这一 技术对工件表面的光洁度没有太多要求, 锈蚀和油污 的影响不大, 所以试件表面不需要经过特别清理。超 声波在工件内部传播, 不一定要将工件拆卸下来, 多 数情况下可以将仪器送到工件旁边直接检测。这样, 可以对有疑问的工件随时监测, 不像以往必须等到大 修返厂解体检修。这种检测方式没有辐射, 也不要加 热, 对人体基本没有危害, 是一种安全的检测方式。

鉴于这是一种无辐射、轻便可视化的检测技术, 不依赖铁磁性, 也不依赖导电性, 在机械、铁路、航 空、航天、船舶制造等领域具有广泛的应用范围。

\section{3 系统结构}

图 2 所示的是超声热波成像技术的结构示意图, 主要由超声激励源、红外热像仪及结构支架、计算机 等组成。其中超声激励源包括超声驱动器、超声换能
器及变幅杆等。超声换能器固定在一移动平台上, 移 动平台由步进电机驱动, 根据工件的大小调节工作距 离, 在移动平台上还安装有压缩气缸, 用于推动超声 换能器并调节其对工件的压力。在压缩气缸与超声换 能器之间有一个压力传感器, 用于控制该压力的大小。 红外热像仪安装在一个可以调节角度与位置的支架 上, 其图像采集与超声激励系统保持同步状态, 以利 于信号的分析。为了提高信噪比, 可以采用多次激励 采集并平均的锁相技术。底座上可对工件进行一定的 夹持, 防止测试时的移动。超声激励装置固定支架上, 方便对试件进行超声激励。红外热像仪安装在超声激 励装置附近位置, 便于采集红外图像, 同时红外热像 仪安装在云平台上方便进行调节。计算机主要用于对 系统控制与红外图像采集以及后期红外图像处理。

\section{2 实验结果}

设备主要由功率超声换能器、红外热成像仪、夹 具、计算机组成。设备采用 $500 \mathrm{~W}$ 超声激励, $20 \mathrm{kHz}$ 激励频率, 单次激励时间不超过 $1 \mathrm{~s}$, 可重复多次激励 以提高信噪比。红外热像仪采用的是制冷热像仪, 分 辨率为 320 pix $\times 256$ pix, 等效噪声温差 (noise equivalent temperature difference, NETD）小于 $20 \mathrm{mK}$, 采集帧频 $60 \mathrm{~Hz}$ 。

裂纹缺陷区域产生的热量与超声波激励加载条 件、加载位置、预紧力大小、试件材料属性、裂纹的 种类和裂纹的形态等因素有关; 因而在实验过程中需 要保证超声波有效注入试件中, 超声枪激励头和试件 接触良好, 这就要求超声枪激励头与试件间施加恒定

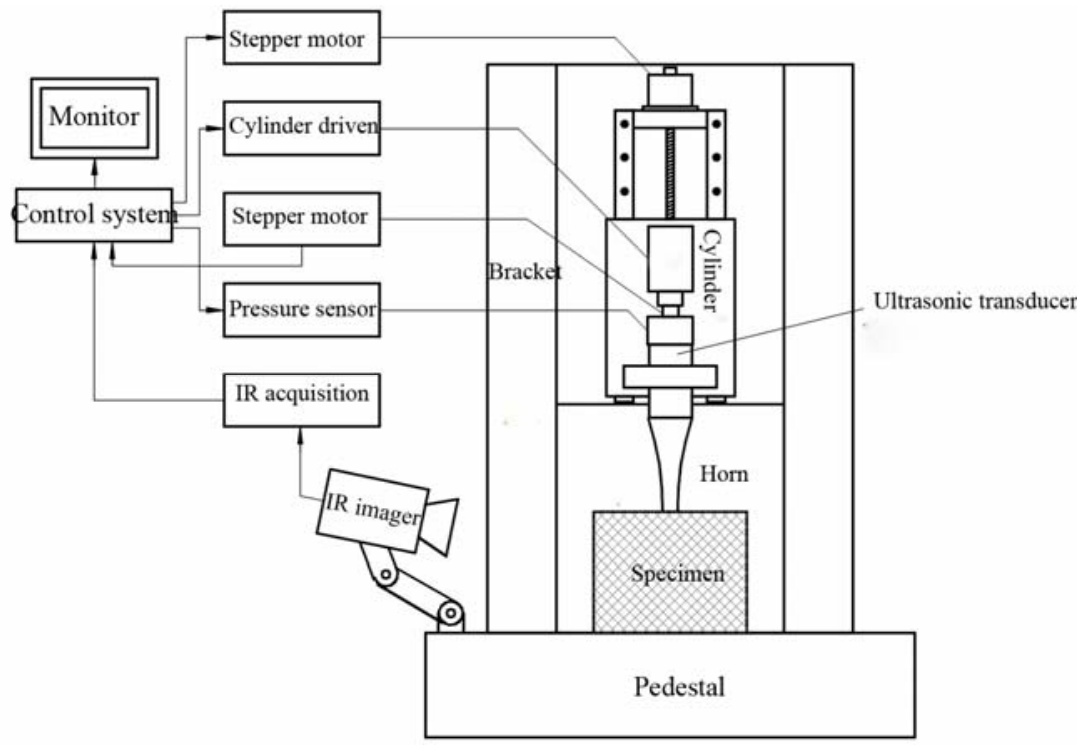

图 2 超声热波成像技术的结构示意图

Fig. 2 Structural sketch of ultrasonic thermal wave imaging technology 
的初始预紧力, 且随着初始预紧力的增加, 耦合到试 件中的能量将越多, 缺陷处产生热量也将越多。超声 枪激励头在 $20 \mathrm{kHz}$ 激励频率下, 与试件激励位置之间 形成间歇性接触，同时在预紧力的作用下，超声波在 试件内部容易形成声混沌现象, 声混沌越混乱, 缺陷 区域产生的温度越高。预紧力的大小与缺陷区域产生 的温度有关, 过小的预紧力不利于超声波耦合进试件 内部, 过大的预紧力导致超声激励头与试件激励位置 无法形成间歇性接触, 同时过大的预紧力容易对试件 造成损伤。

图 3 和图 4 为机车钩舌试件, 机车钩舌重 $35 \mathrm{~kg}$, (a)为超声激励位置点光学照片, (b) 为缺陷位置点光学 照片, (c)为检测缺陷结果图像; 图 3(c)中可看到明显 $\mathrm{L}$ 型裂纹，图 4(c)中可检测出裂纹。

实验检测过程中发现超声激励后内部裂纹缺陷 对应的表面温度最高, 热波信号最强, 红外图像中表 现为亮斑, 无裂纹区域对应的表面温度没有变化。预 紧力在实验过程中非常关键, 通过多次预紧力的调 节, 预紧力在 $200 \mathrm{~N}$ 左右时, 缺陷位置温度最高; 为 了更好地检测出钩舌试件裂纹缺陷, 需要激励不同区 域, 以达到完全覆盖整个试件。在超声激励作用下, 试件中会出现强烈的非线性振动现象, 包括超谐波振
动、次谐波振动、准次谐波振动、混沌等; 超声激励 过程中的非线性振动变现为即使能量较低的情况下 也会取得较好的检测效果，声混沌产生与预紧力有 关，不依赖于缺陷存在。待测试件的强非线性振动现 象能够大大增加超声红外热波成像技术对缺陷的检 测能力。

红外热波信号产生的强弱与几个参数密切相关, 在实验过程中需要摸索出实验参数。超声换能器与工 件之间的压力: 这对热波信号的激励十分重要，压力 过小声波耦合效率不高, 但过高会对换能器的压力太 大，使其不能正常工作，根据前期实验的结果，这个 压力一般控制在 $200 \mathrm{~N}$ 附近; 超声激励的频率：由于 特定频率在工件内部可能产生驻波，因此如果裂纹处 在波谷处则无法激励热波信号，故而采用可调频高功 率超声驱动器, 其频率范围可在 $18 \sim 22 \mathrm{kHz}$ 之间调 谐, 功率输出在 $500 \sim 1000 \mathrm{~W}$ 左右; 超声换能器与工 件接触位置: 超声变幅杆与工件直接接触, 触点的位 置很重要, 有些接触部位可能在裂纹的地方产生声场 的盲区而无法激励热波信号。该技术可以让工件相对 超声变幅杆进行各种平移和转动, 而热像仪也将随之 变动。超声耦合的方式: 超声变幅杆与工件接触时如 果耦合不好则能量不能有效传递，同时对一些表面有

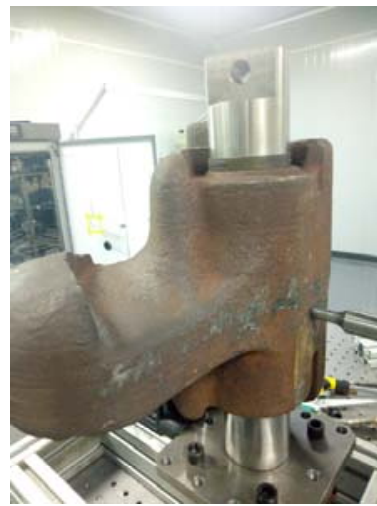

(a) 超声激励位置

(a) Ultrasonic excitation location

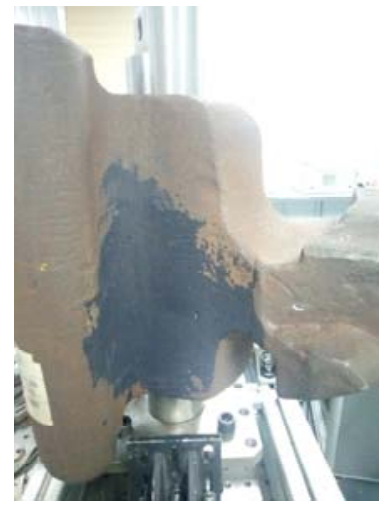

(b) 缺陷位置

(b) Defect location

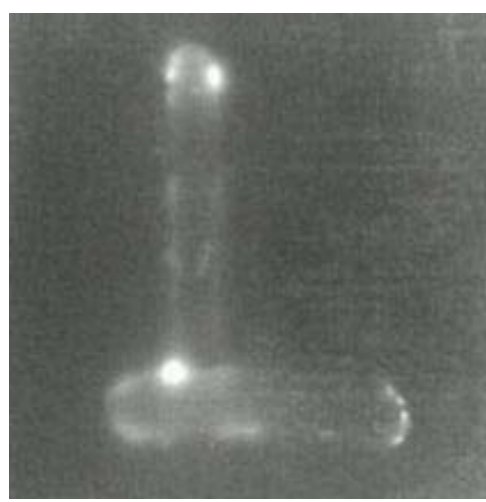

(c) 检测缺陷结果

(c) Defect detection results

图 3 钩舌试件 1 Fig. 3 Coupler tongue specimen 1

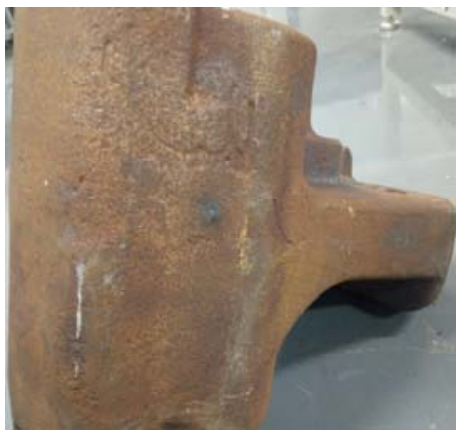

(a) 超声激励位置

(a) Ultrasonic excitation location

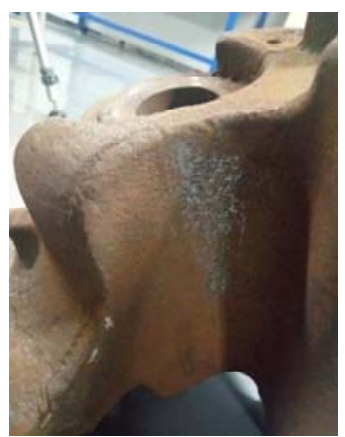

(b) 缺陷位置

(b) Defect location

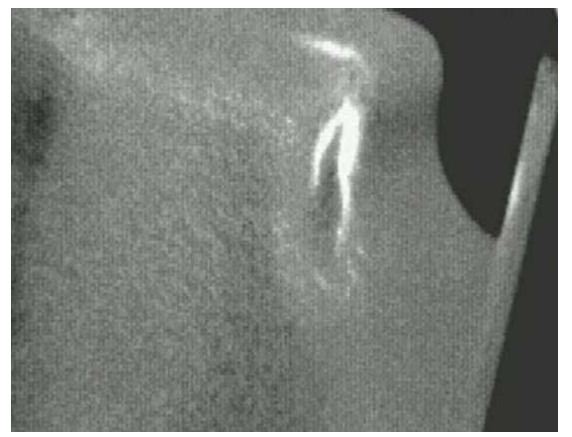

(c) 检测缺陷结果

(c) Defect detection results

图 4 钩舌试件 2 Fig. 4 Coupler tongue specimen 2 
一定要求的工件直接接触可能造成损伤, 所以需要在 超声变幅杆与工件之间使用耦合材料, 这些材料需要 有一定的柔性, 具有很强的韧性。可以采用化纤祄底 的胶带、高强度纸张等材料。

\section{3 结语}

超声热波成像技术是一种新兴检测技术, 可以用 于对列车车头、车架、轴承、钩头、车钩等零部件进 行检测, 可以有效提高列车运营的安全性, 减小事故 发生概率, 为企业提供更为有效的安全保障, 更为有 效地减小企业运营成本。相对于常规检测手段, 有显 著的技术优势, 具有明显的推广价值。可以有效解决 目前一些零部件裂纹缺陷无法有效检测的难题。本文 设计开发了一套超声热波成像无损检测系统, 系统可 以很好地实现超声枪与试件的耦合, 最后通过该系统 对两个铁路机车钩舌试件进行了检测, 检测出了两个 铁路机车钩舌的裂纹缺陷, 验证了该系统对机车钩舌 裂纹的检测能力, 为这些部件的质量控制及维修工艺 提供参考依据。

\section{参考文献:}

[1] 杜云慧, 张励忠, 邢书明, 等. 钩舌常见裂纹形式与产生机理研究 [J]. 铁道机车车辆, 2003, 23(3): 21-23.

DU Yunhui, ZHANG Lizhong, et al. XING Shuming et al. Study on Formation and Mechanism of Cracks in Hook-head[J]. Railway Locomotive \& Car, 2003, 23(3) : 21-23.

[2] 李晓慧, 谢基龙. 重载列车 $\mathrm{E}$ 级钢钩舌疲劳裂纹扩展条件及寿命预测 [J]. 北京交通大学学报, 2006, 30(4): 102-104.

LI Xiaohui, XIE Jilong. Prediction of Fatigue Crack Growth Condition and Lifetime on Coupler Guard Arm Area of E Grade Steel of Burden Strain[J]. Journal of Beijing Jiaotong University, 2006, 30(4):102-104.

[3] 张继周, 突玉香. 钩舌裂纹产生机理的分析与探讨 [J]. 河南建材, 2011, 3(1): 137-138.

ZHANG Jizhou, DOU Yuxiang. Analysis and discussion on crack generation mechanism of hook tongue[J]. Henan Building Materials, 2011, 3(1): 137-138.

[4] 杜云慧, 张励忠, 邢书明, 等. 钩舌常见失效形式与机理[J]. 金属热处 理, 2003, 28(6): 48-52.

DU Yunhui, ZHANG Lizhong, XING Shuming, et al. Failure forms of the hook-head and its mechanism[J]. Heat Treatment of Metals, 2003, 28(6): 48-52.

[5] CHEN Zhaojiang, ZHENG Jiang, ZHANG Shuyi, et al. Finite element modeling of heating phenomena of cracks excited by high-intensity ultrasonic pulses[J]. CHINESE PHYSICS B, 2010, 19(11): 682-694 .

[6] 刘慧. 超声红外锁相热像无损检测技术的研究[D]. 哈尔滨: 哈尔滨工 业大学, 2010.

LIU Hui. Research on the Technology of Ultrasonic Infrared Phase-locked Thermography Nondestructive Testing[D]. Harbin: Harbin University of Technology, 2010.

[7] 陈大鹏. 超声脉冲及超声脉冲相位热像技术用于无损检测的实验研究
[D]. 北京: 首都师范大学, 2009 .

CHEN Dapeng. Experimental Study on Non-destructive Testing Using Ultrasound Pulse and Phase Thermography[D]. Beijing: Capital Normal University, 2009.

[8] 冯辅周, 张超省, 袁建, 等. 超声红外热像技术中裂纹的识别和重构 [J]. 无损检测, 2011, 33(11): 17-20.

FENG Fuzhou, ZHANG Chaosheng, YUAN Jian, et al. Identification and reconstrution of cracks in ultrasonic IR imaging[J]. NondestructiveTesting, 2011, 33(11): 17-20.

[9] ZHENG Kai, ZHANG Shuyi, CHEN Zhaojiang. Anomalous subharmonics excited by intensive ultrasonic pulses with a single frequency[J]. Applied Physics Letters, 2008, 9(2): 22-25.

[10] 张炜, 罗文源, 王冬冬, 等. 基于超声热波技术的复合材料冲击损伤 检测研究[J]. 固体火箭技术, 2013, 36(6): 836-841.

ZHANG Wei, LUO Wenyuan, WANG Dongdong, et al. Impact damage detection of domposites based on ultrasonic thermography technique[J]. Journal of Solid Rocket Technology, 2013, 36(6): 836-841.

[11] 徐长航, 谢静, 周乃望, 等. 新型超声红外热像无损检测实验系统研 制[J]. 实验技术与管理, 2016, 33(10): 71-75.

XU Changhang, XIE Jing, ZHOU Naiwang, et al. Development of novel experimental system for structural non-destructive testing based on ultrasonic tehermography[J]. Experimental Technology and Management, 2016, 33(10): 71-75.

[12] 秦雷, 刘俊岩, 龚金龙, 等. 超声红外锁相热像技术检测金属板材表 面裂纹 [J]. 红外与激光工程, 2013, 42(5): 1123-1129.

QIN Lei, LIU Junyan, GONG Jinlong, et al. Testing surface crack defects of sheetmetal with ultrasonic lock-in thermography[J]. Infrared and Laser Engineering, 2013, 42(5): 1123-1129.

[13] 金国锋, 张炜, 宋远佳, 等. 含曲率结构裂纹的超声红外热波检测数 值仿真 [J]. 科学技术与工程, 2013, 13(3): 776-779.

JIN Guofeng, ZHANG Wei, SONG Yuanjia, et al. Numerical simulation for ultrasonic infrared thermal wave detecting of curvature structural crack[J]. Science and Technology and Engineering, 2013, 13(3): 776-779.

[14] 冯辅周, 张超省, 江鹏程, 等. 基于超声红外热像技术的装甲装备缺 陷检测系统的设计与实现[J]. 光电子技术, 2012, 32(3): 170-174.

FENG Fuzhou, ZHANG Chaosheng, JIANG Pengchen, et al. Design and implement of defect detection system for armored equipments based on ultrasonic infrared thermography[J]. Optoelectronic Technology, 2012, 32(3): 170-174.

[15] 冯辅周, 张超省, 张丽霞, 等. 超声红外热波技术的研究现状[J]. 无 损检测, 2012, 34(9): 46-50.

FENG Fuzhou, ZHANG Chaosheng, ZHANG Lixia, et al. Research situation of ultrasonic infrared thermal wave technology[J]. NondestructiveTesting, 2012, 34(9): 46-50.

[16] 陈大鹏, 张存林, 李晓丽, 等. 超声热红外技术在无损检测领域中的 应用[J]. 激光与红外, 2008, 38(8): 778-780.

CHEN Dapeng, ZHANG Cunlin, LI Xiaoli, et al. Ultrasonic infrared thermal wave technology and its applications in nondestrutive evalution[J]. Laser and Infrared, 2008, 38(8): 778-780. 